\title{
Prognostic value of the immediate response to surfactant
}

\author{
J Kuint, B Reichman, L Neumann, E S Shinwell
}

\begin{abstract}
Aim-To evaluate the association between the immediate response to surfactant treatment and morbidity and mortality in infants with severe respiratory distress syndrome.

Methods-The response to surfactant was defined as the difference in the arterial:alveolar $(\delta$ a:A) ratio before and one hour after the first surfactant dose. Measurements were obtained from 253 Israeli infants participating in the multicentre Curosurf 4 trial of surfactant replacement therapy.

Results-Delta a:A ratios ranged from -0.115 to 0.8 and were significantly related to both birth weight and gestational age. Among infants weighing 1001-1500 g mortality decreased from $40 \%$ among very bad responders to zero among good responders. The incidence of pneumothorax decreased with better response. Logistic regression analysis showed a hierarchy of predictive power for mortality: birth weight or gestational age; immediate response to surfactant; severity of initial disease.

Conclusion-The immediate response to surfactant treatment is a significant prognostic indicator for mortality and morbidity.
\end{abstract}

(Arch Dis Child 1994; 71: F170-F173)

Numerous clinical trials have shown that surfactant replacement therapy is highly effective for neonatal respiratory distress syndrome (RDS). ${ }^{1}$ A dramatic improvement in oxygenation occurred in infants treated with surfactant. $^{2-4}$ Mortality decreased by about $40 \%$ in most of the reported studies. ${ }^{2-4}$ The incidence of pneumothorax fell to less than $10 \%$ and length of hospital stay was shortened. ${ }^{2-4}$ However, the incidence of bronchopulmonary dysplasia has remained unchanged despite the use of surfactant, although the number of survivors without has steadily increased during recent years. ${ }^{5}$

According to various reports of several different surfactant preparations, $6-24 \%$ of infants respond poorly to surfactant. ${ }^{6-10} \mathrm{We}$ hypothesised that poor response to surfactant may be an indicator of poor prognosis in terms of morbidity and mortality. The aim of this study was to evaluate the association between the initial response to surfactant and neonatal morbidity and mortality and to compare poor response with other recognised prognostic indicators, such as birth weight, gestational age, and severity of initial disease as reflected in Apgar score, highest $\mathrm{FiO}_{2}$, and lowest $\mathrm{pH}$ in the first 12 hours of life.

\section{Methods}

The infants studied were enrolled in a multicentre, prospective, randomised clinical trial of surfactant replacement therapy (Curosurf 4). ${ }^{11}$ We compared two different dose protocols of porcine surfactant (Curosurf; Chiesi Farmaceutici, Parma, Italy). Curosurf is a natural surfactant isolated from minced pig lungs by a combination of washing, chloroform-methanol extraction, and liquid-gel chromatography. ${ }^{12}$ The high dose protocol consisted of a loading dose of $200 \mathrm{mg} / \mathrm{kg}$, followed by up to four subsequent doses of $100 \mathrm{mg} / \mathrm{kg}$; the low dose protocol comprised three doses of $100 \mathrm{mg} / \mathrm{kg}$. Eighty one centres throughout Europe contributed to the study. This analysis relates to the infants from the eight Israeli centres in the study.

\section{DATA COLLECTION}

The baseline data collected on each infant included maternal medical and obstetric history, antenatal steroid treatment, mode of delivery, duration of rupture of membranes, single or multiple pregnancy, fetal presentation, Apgar score and sex. Data relating to RDS included ventilator settings before and after treatment and blood gases for all surfactant doses. Outcome was assessed by days of mechanical ventilation, days in $\mathrm{O}_{2}$ (above and below $\mathrm{FIO}_{2}=0.4$ ), major morbidity, such as air leak complications, sepsis, intraventricular haemorrhage, periventricular leucomalacia, patent ductus arteriosus, necrotising enterocolitis, and retinopathy of prematurity.

Immediate response was defined as the difference between the arterial:alveolar (a:A) ratio immediately before and one hour after the first Curosurf dose ( $\delta$ a:A ratio). The a:A ratio was calculated according to the following formula:

$$
\mathrm{Pa} / \mathrm{AO}_{2}=\mathrm{PaO}_{2} /\left(713 \times \mathrm{FIO}_{2}\right)-\mathrm{PaCO}_{2} \text {. }
$$

The $\delta$ a:A ratio was calculated for all 253 infants and arranged in ascending order (figure). The range of $\delta$ a:A ratio was from -0.115 to $0 \cdot 8$. The values obtained were divided into four quartiles, arbitrarily termed very bad, bad, fair and good response for analysis (figure).

\section{DATA ANALYSIS}

The influence of the factors described on response type, major morbidity, and mortality 


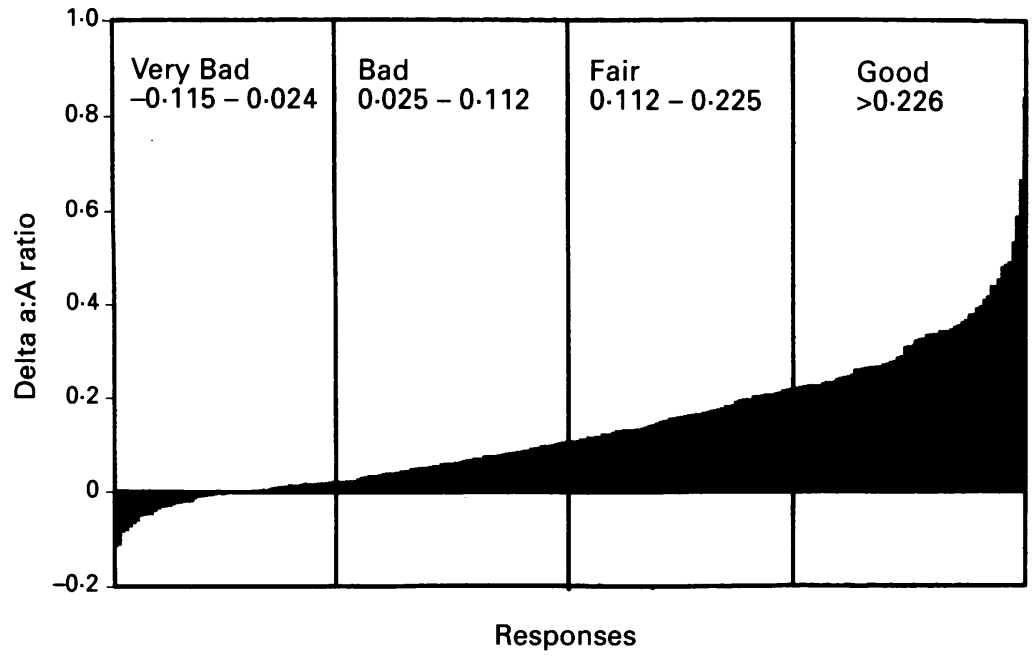

Response to the first dose of surfactant treatment as measured by the $\delta$ a: $A$ ratio in 253 infants. Each column represents a single infant. Response was arbitrarily divided into quartiles and the range of values in each quartile is shown under the assigned name.

was assessed using $\chi^{2}$ and Fisher's exact tests for qualitative variables. Pearson's correlation coefficients were computed to assess the association between continuous variables. The independent effect of response type and birth weight on mortality was assessed by trend analysis. Multiple logistic regression analysis was used to assess the relative predictive power of each of the following independent variables (birth weight; gestational age; highest $\mathrm{FIO}_{2}$ and lowest $\mathrm{pH}$ in the first 12 hours; Apgar score at five minutes; dose assignment and response type) on dependent variables, including mortality, days of mechanical ventilation, days in $\mathrm{O}_{2}$, length of stay, pneumothorax, other air leak, intraventricular haemorrhage and periventricular leucomalacia.

Preliminary analysis failed to demonstrate any significant differences in response type or outcome in terms of morbidity and mortality between the two dose protocols and therefore, for the purposes of subsequent analysis, the entire population was studied as a whole.

\section{Results}

Two hundred and fifty three infants were included in the analysis. Mean (SD) birth weight was 1380 (507) $\mathrm{g}$ and mean gestational age was $29(3 \cdot 6)$ weeks. Male:female ratio was 1.33:1. Mean Apgar score was $5 \cdot 7$ at one minute and $8 \cdot 2$ at five minutes. Twenty one point four per cent were treated with antenatal steroids and prolonged rupture of membranes was present in $18 \%$. Fifty per cent of the infants had been delivered by caesarean section.

Table 1 Mortality in relation to birth weight and response type as defined by $\delta$ a:A ratio (as shown in figure)

\begin{tabular}{|c|c|c|c|c|c|}
\hline \multirow[b]{2}{*}{$\begin{array}{l}\text { Birth weight } \\
(g)\end{array}$} & \multicolumn{5}{|l|}{ Response } \\
\hline & $\begin{array}{l}\text { Very bad } \\
n(\%)\end{array}$ & $\begin{array}{l}\text { Bad } \\
n(\%)\end{array}$ & $\begin{array}{l}\text { Fair } \\
n(\%)\end{array}$ & $\begin{array}{l}\text { Good } \\
n(\%)\end{array}$ & $\begin{array}{l}\text { Total } \\
n(\%)\end{array}$ \\
\hline $\begin{array}{l}<750 \\
751-1000 \\
1001-1500 \\
>1500 \\
\text { Total }\end{array}$ & $\begin{array}{c}10 / 10(100) \\
15 / 25(60) \\
8 / 20(40) \\
2 / 9(22) \\
35 / 64(53)\end{array}$ & $\begin{array}{r}8 / 12(67) \\
8 / 14(57) \\
3 / 15(20) \\
3 / 22(14) \\
22 / 63(34)\end{array}$ & $\begin{array}{c}6 / 7(86) \\
4 / 11(36) \\
2 / 23(9) \\
0 / 22(0) \\
12 / 63(19)\end{array}$ & $\begin{array}{c}7 / 8(88) \\
4 / 10(40) \\
0 / 15(0) \\
1 / 30(3) \\
12 / 63(18)\end{array}$ & $\begin{array}{c}31 / 37(84) \\
31 / 60(52) \\
13 / 73(18) \\
6 / 83(7) \\
81 / 253(32)\end{array}$ \\
\hline
\end{tabular}

Table 2 Patient characteristics according to response type ${ }^{\star}$

\begin{tabular}{|c|c|c|c|c|}
\hline \multirow[b]{2}{*}{ Response } & \multicolumn{2}{|c|}{$\begin{array}{l}\text { Mean Apgar } \\
\text { score (minutes) }\end{array}$} & \multirow{2}{*}{$\begin{array}{l}\text { Gestational } \\
\text { age (weeks) }\end{array}$} & \multirow{2}{*}{$\begin{array}{l}\text { Birth weight } \\
\text { (g) }\end{array}$} \\
\hline & 1 & 5 & & \\
\hline $\begin{array}{l}\text { Very bad } \\
\text { Bad } \\
\text { Fair } \\
\text { Good }\end{array}$ & $\begin{array}{l}4 \cdot 4 \\
5 \cdot 4 \\
6 \cdot 3 \\
5 \cdot 8\end{array}$ & $\begin{array}{l}7 \cdot 2 \\
8 \cdot 1 \\
8 \cdot 3 \\
8 \cdot 3\end{array}$ & $\begin{array}{l}27 \cdot 8(2 \cdot 6) \\
28 \cdot 1(3 \cdot 1) \\
28 \cdot 8(3 \cdot 2) \\
30 \cdot 1(3 \cdot 2)\end{array}$ & $\begin{array}{l}1073(380) \\
1247(532) \\
1441(880) \\
1484(616)\end{array}$ \\
\hline
\end{tabular}

^Results expressed as mean (SD).

FACTORS INFLUENCING MORTALITY

Mortality in the 'very bad' response group was $53 \%, 34 \%$ in the bad group, $19 \%$ in the fair group, and $18 \%$ in the good group. Mortality was $84 \%, 52 \%, 18 \%$, and $7 \%$ in the birth weight groups at $<751 \mathrm{~g}, 751-1000 \mathrm{~g}$, $1001-1500 \mathrm{~g}$, and $>1500 \mathrm{~g}$, respectively. Analysis of mortality by response type for each weight group is shown in table 1. A significant correlation between mortality and response type was seen in the 1001-1500 $\mathrm{g}$ groups $(p=0.006)$. A similar though not significant trend was seen in the other weight groups.

\section{FACTORS INFLUENCING RESPONSE TYPE}

Mean (SD) birth weight in the 'very bad' responders was 1073 (380) g compared with 1247 (532) g, 1441 (880) g, and 1484 (616) g in the 'bad', 'fair', and 'good' response groups, respectively $(\mathrm{p}<0.01)$. Mean (SD) gestational age was $27 \cdot 8(2 \cdot 6)$ weeks in the 'very bad' responders compared with $29 \cdot 1$ $(3 \cdot 1), 29 \cdot 9(3 \cdot 2)$, and $30 \cdot 1(3 \cdot 2)$ weeks in the 'bad', 'fair', and 'good' groups, respectively $(p<0.01)$. Apgar scores at one and five minutes did not differ significantly between the groups.

\section{RELATION BETWEEN RESPONSE TYPE AND} MORBIDITY PATTERNS

The incidence of pneumothorax according to response type was $20 \%, 16 \%, 5 \%$, and $3 \%$ in the 'very bad', 'bad', 'fair', and 'good' groups, respectively $(p=0.023)$. No significant correlation was found between response type and pulmonary haemorrhage, intraventricular haemorrhage, periventricular leucomalacia, retinopathy of prematurity, necrotising enterocolitis, sepsis, apnoea, pneumonia, and seizures.

ANALYSIS OF RELATIVE PREDICTIVE POWER OF FACTORS INFLUENCING MORTALITY

Stepwise multiple logistic regression analysis of the above mentioned independent variables showed that birth weight had the most profound effect on mortality $(p=0.00001)$. As the correlation between birth weight and gestational age was extremely high $(\mathrm{r}=0 \cdot 88)$ only birth weight was included in the analysis. Similarly, highest $\mathrm{FIO}_{2}$ and lowest $\mathrm{pH}$ were inversely correlated and therefore only highest $\mathrm{FIO}_{2}$ was included. Response type was the second most powerful indicator $(p=0.0047)$ and highest $\mathrm{FIO}_{2}$ in the first 12 hours was of borderline significance $(p=0 \cdot 065)$. 


\section{Discussion}

In this study the initial response to surfactant administration, as measured by $\delta$ a:A ratio, was a predictor of mortality. A higher $\delta$ a:A ratio was clearly associated with a decrease in mortality, adding this early parameter to other, already established prognostic factors such as birth weight, gestational age, and severity of initial disease, as reflected by initial $\mathrm{pH}$ and supplemental oxygen requirement. ${ }^{13}$

Previous studies suggest that almost a third of all infants treated with surfactant are 'poor' responders. ${ }^{6-10}$ Different definitions of response and different surfactant agents may, in part, explain the wide range of reported incidence of poor response. Segerer et al defined three types of response to Curosurf using the rate and duration of the reduction in oxygen requirement within the first 60 hours after treatment. ${ }^{6}$ Fourteen per cent of their infants responded poorly. Charon et al, using Surfactant TA, defined three types of response according to the a: $\mathrm{APO}_{2}$ over the first 48 hours of treatment and found that $24 \%$ had an 'absent' response. ${ }^{7}$ The Collaborative European Multicenter Study Group (Curosurf) found a $24 \%$ poor response using a combination of change in a:A ratio and mortality. ${ }^{8}$ Fujiwara et al reported $6 \%$ of poor response $^{9}$ and Hallman reported $8 \%$ using other definitions. ${ }^{10}$ Recently, Hamvas et al, using Exosurf, defined unresponsiveness as a decline in oxygen index of less than $25 \%$ within six hours of surfactant administration. ${ }^{14}$ This broad definition resulted in a $50 \%$ poor response.

We have shown that an absent or poor response to the first dose of surfactant has significant prognostic value. Most of the studies reported used definitions based on parameters measured a few hours or even days after surfactant administration and most demonstrated a significant prognostic value for poor response on mortality. The only report evaluating short term response, 30 minutes after surfactant administration, was the Collaborative European Multicenter Study Group which was unable to demonstrate an influence of the response type on mortality. The difference in rate of response to natural and synthetic surfactants (minutes with natural and hours with synthetic) is relevant when defining response criteria. Using Curosurf, we preferred a rapid response (one hour) to minimise the influence of any other therapeutic interventions which may mask the effect of surfactant.

Patterson and Halliday used a statistical model consisting of gestational age, Apgar score at five minutes, and the presence or absence of respiratory distress for prediction of outcome. ${ }^{15}$ They correctly predicted outcome for $94 \%$ of survivors but for only $53 \%$ of deaths. Adding $\delta$ a:A ratio to their model might improve the predictive value, particularly for mortality.

The prognostic value of response was also evaluated in relation to morbidity. We found that a low $\delta$ a:A ratio was associated with an increased incidence of pneumothorax.
Bronchopulmonary dysplasia, represented as days with $\mathrm{FIO}_{2}$ of $\geqslant 0 \cdot 4$, or all days in supplemental oxygen, was more common in infants with a poor response, although this did not reach significance $(p=0.058)$. Response type did not influence the incidence of intraventricular haemorrhage, periventricular leucomalacia, retinopathy of prematurity, necrotising enterocolitis or apnoea of prematurity. Hamvas similarly reported a higher incidence of pneumothorax and pulmonary interstitial emphysema in the 'non-response' group. ${ }^{14}$ Bronchopulmonary dysplasia was more common in the 'response' group, but this did not reach significance. Charon in his study and the Collaborative European Multicenter Study Group found the same effect of response on pneumothorax and pulmonary interstitial emphysema but could not demonstrate an influence on bronchopulmonary dysplasia. These studies support our findings which show poor response as an important predictor for mortality and early pulmonary morbidity.

Birth asphyxia and perinatal infection can interfere with surfactant production and action. ${ }^{16}$ Unlike Segerer, we could not show that low Apgar score or infection were related to response type. Segerer suggested that infection has a negative impact on response. ${ }^{6}$ It must be emphasised that only confirmed sepsis (positive bacterial blood culture) was taken into consideration, though many infants had important clinical signs of sepsis such as hypotension, poor perfusion, leucopenia, and thrombocytopenia but negative blood cultures. Others had been partially treated because of prenatal maternal antibiotic treatment and hence the number of infants with any kind of infection (bacterial, viral, chlamydial, mycoplasmal or fungal) acquired perinatally is unknown. Prevention of infection may be extremely important in increasing response rate and thus improving survival.

Appreciation of the prognostic importance of the immediate response to surfactant may help us identify a high risk group of premature infants in whom new or more aggressive treatment may improve outcome and conversely may reduce the necessity for potentially harmful interventions in those infants who respond appropriately.

1 Fujiwara T, Chida S, Watabe Y, et al. Artificial surfactan therapy in hyaline membrane disease. Lancet 1980; 55-9.

2 Hallman M, Merritt TA, Jarvengaa AL, et al. Exogenous human surfactant for treatment of severe respiratory distress syndrome: A randomized prospective clinical trial. 7 Pediatr 1985; 106: 963-9.

3 Phibbs RH, Ballard RA, Clements JA, et al. Initial clinica trial of EXOSURF, a protein-free synthetic surfactant, for ( disease. Pediatrics 1991;

4 Long W, Thompson T, Sundell $H$, et al. Effects of two rescue doses of a synthetic surfactant on mortality rate rescue doses of a synthetic surfactant on mortality rate and survival without bronchopulmonary dysplasia in 700 to 1350 gram infants with respir

5 Hudak BB, Egan EA. Impact of lung surfactant on the development of bronchopulmonary dysplasia. Clin Perinatol 1992; 19: 591-602.

6 Segerer H, Stevens P, Schadow B, et al. Surfactant substitution in ventilated very low birth weight infants: factors related to response types. Pediatr Res 1991; 30 591-6.

7 Charon A, Taeusch HW, Fitzgibbon C, et al. Factors associated with surfactant treatment response in infants with severe respiratory distress syndrome. Pediatrics 1989 83: $348-54$ 
8 Collaborative European Multicentre Study Group. Factors influencing the clinical response to surfactant replacement therapy in babies with severe respiratory distress syntherapy in babies with severe respira

9 Fujiwara T, Konishi M, Chida S, et al. Factors affecting the response to a postnatal single dose of a reconstituted bovine surfactant (Surfactant TA). In: Lachmann B, ed. Surfactant replacement therapy in neonatal and adult respiratory distress syndrome. Berlin: Springer-Verlag, 1988: 99-107.

10 Hallman M, Pohjavuori M, Bry K, et al. Human surfactant from amniotic fluid in the treatment of respiratory distress syndrome. In: Lachman $B$, ed Surfactant ed. Surfactant replacement therapy in neonatal and adult respiratory distress $136-42$.

11 Halliday HL Tarnow-Mordi W, Corcoran D, Patterson CC. A multicentre, randomized trial comparing high and low dose regimens for the treatment of respiratory distress syndrome (The Curosurf 4 trial). Arch Dis Child 1993; 69: 276-80.

12 Collaborative European Multicenter Study Group. Surfactant replacement therapy for severe respiratory distress syndrome: An international randomized controlled trial. Pediatrics 1988; 82: 683-91.

3 The CRIB (Clinical risk index for babies) score: a tool for assessing initial neonatal risk and comparing performance of neonatal intensive care units. The international neonatal network. Lancet 1993; 342: 189-93.

14 Hamvas A, Devine T, Cole FS. Surfactant therapy failure identifies infants at risk for pulmonary mortality. Am $f$ Dis Child 1993; 147: 665-81.

15 Patterson CC Halliday HI. Prediction of outcome after delivery for the very low birth weight $(<1500 \mathrm{~g})$ after delivery for the very low birth weight
infant. Pediatr Perinat Epidemiol 1988; 2: 221-8.

16 Hessler JR, Montilla G, Kirkpatrick BV, et al. Asphyxia and hyaline membrane disease in neonatal monkeys. Am $\mathcal{f}$ Perinatol 1985; 2: 101-7. 